\title{
Reduced retinoic acid synthesis accelerates prophase I and follicle activation
}

\author{
Roseanne Rosario, Hazel L Stewart, Emily Walshe and Richard A Anderson \\ MRC Centre for Reproductive Health, Queen's Medical Research Institute, University of Edinburgh, Edinburgh, UK \\ Correspondence should be addressed to R Rosario; Email: roseanne.rosario@ed.ac.uk
}

\begin{abstract}
In female mammals, reproductive potential is determined during fetal life by the formation of a non-renewable pool of primordial follicles. Initiation of meiosis is one of the defining features of germ cell differentiation and is well established to commence in response to retinoic acid. WIN 18,446 inhibits the conversion of retinol to retinoic acid, and therefore it was used to explore the impact of reduced retinoic acid synthesis on meiotic progression and thus germ cell development and subsequent primordial follicle formation. e13.5 mouse fetal ovaries were cultured in vitro and treated with WIN 18,446 for the first 3 days of a total of up to 12 days. Doses as low as $0.01 \mu \mathrm{M}$ reduced transcript levels of the retinoic acid response genes Stra8 and Rar $\beta$ without affecting germ cell number. Higher doses resulted in germ cell loss, rescued with the addition of retinoic acid. WIN 18,446 significantly accelerated the progression of prophase I; this was seen as early as $48 \mathrm{~h}$ post treatment using meiotic chromosome spreads and was still evident after 12 days of culture using Tra98/Msy2 immunostaining. Furthermore, ovaries treated with WIN 18,446 at e13.5 but not at P0 had a higher proportion of growing follicles compared to vehicle controls, thus showing evidence of increased follicle activation. These data therefore indicate that retinoic acid is not necessary for meiotic progression but may have a role in the regulation of its progression and germ cell survival at that time and provide evidence for a link between meiotic arrest and follicle growth initiation. Reproduction (2020) $160331-341$
\end{abstract}

\section{Introduction}

Female reproductive senescence results from the depletion of a finite ovarian follicle pool that is assembled during fetal life. These follicles are produced from primordial germ cells which transition to oocytes as they undergo loss of pluripotency, proliferate with incomplete cytokinesis forming germ cell nests, and enter into meiosis. The initiation of meiosis is one of the defining features of germ cell differentiation and occurs in fetal life in female mammals. Although meiosis is comprised of two rounds of cell division, only prophase of meiosis I occurs during fetal oogenesis, with arrest occurring before completion of the first division.

In fetal ovaries, the first signs of meiosis initiation are apparent between embryonic day 12.5 and 13.5 in mice (Adams \& McLaren 2002) and from gestational week 12 in humans (Gondos et al. 1986, Le Bouffant et al. 2010). Current understanding of meiosis initiation and regulation is primarily derived from studies in mice, and it is generally accepted that the onset of meiosis depends on exposure to retinoic acid. Indeed, early studies demonstrated that long-term vitamin A deprivation (the precursor in retinoic acid synthesis) caused spermatogenic arrest in rodents (Huang \& Hembree 1979, Griswold et al. 1989), while more recent studies have shown that maternal deficiency of vitamin
A resulted in female offspring with undifferentiated germ cells which had failed to initiate meiosis, which could be rescued in pregnant vitamin A-deficient rats through administration of retinoic acid ( $\mathrm{Li} \&$ Clagett-Dame 2009). Retinoic acid is produced by the mesonephros in rodents (Bowles et al. 2006, Koubova et al. 2006) but probably by the fetal ovary itself in humans (Le Bouffant et al. 2010, Childs et al. 2011, Bowles et al. 2016, Frydman et al. 2017), through the reversible conversion of retinol (dietary vitamin A) to retinaldehyde by alcohol dehydrogenases. Retinaldehyde is then irreversibly oxidised by retinaldehyde dehydrogenases (RALDHs) 1, 2, and 3 (Griswold et al. 2012). In the fetal testis, endogenous retinoic acid is cleared by CYP26B1, ensuring that the onset of meiosis in male germ cells is delayed until after birth (reviewed in Feng et al. 2014). Due to its lipophilic nature, retinoic acid easily diffuses through cell membranes to interact with heterodimerised retinoic acid receptors $(\operatorname{Rar} \alpha, \beta$, and $\gamma)$, which are found bound to retinoic acid-responsive elements in regulatory regions of target genes. In the presence of retinoic acid, these receptors act to enhance the transcription of genes that are otherwise silenced. Retinoic acid mediates the transcription and expression of the meiotic factor Stra8 (stimulated by retinoic acid gene 8 ), which is required for pre-meiotic DNA replication (Baltus et al. 2006), and 
governs the subsequent events of meiotic prophase (Lin et al. 2008) through the upregulation of DNA meiotic recombinase Dmc1 (Menke et al. 2003). In female mouse germ cells, Rar and Stra8 expression have been shown to coincide with retinoic acid production and the onset of meiosis at approximately embryonic day 13.5 (Morita \& Tilly 1999, Dokshin et al. 2013).

Following diplotene, nests of interconnected germ cells break down to release individual oocytes which associate with somatic pre-granulosa cells forming primordial follicles; this takes place during early postnatal life in rodents (Pepling \& Spradling 2001) and in humans begins around 15 weeks gestation (Motta et al. 1997, Bendsen et al. 2006). These primordial follicles remain in a dormant state within the ovarian cortex until they receive signals for activation and are recruited to grow. Whether germ cell nest breakdown and primordial follicle formation are developmentally linked to meiotic progression remains poorly understood. Deletion of genes responsible for the initial stages of prophase, including Dazl, Spo11, Dmc1, Atm, Msh4 and Msh5, result in loss of oocytes and an inability to form follicles, precluding analysis (Pepling 2006, Edson et al. 2009). Conversely, the ovaries of Stra8 ${ }^{-/-}$mice contain oocytelike cells and follicular structures, despite being unable to initiate meiosis (Baltus et al. 2006, Dokshin et al. 2013), and depletion of Sycp 1 actually accelerates the onset of diplotene and results in primordial follicles being assembled earlier and in greater numbers than in control ovaries (Paredes et al. 2005). Thus the conflicting evidence surrounding the intricate relationship between meiotic progression, and particularly diplotene arrest, and primordial follicle formation needs to be explored further in physiological conditions.

WIN 18,446 belongs to the bis-dichlororacetyldiamines (BDAD) family of compounds and can inhibit the enzymatic activity of at least one of the aldehyde dehydrogenases, thus preventing the conversion of retinol to retinoic acid (Amory et al. 2011). It was initially investigated as a non-hormonal male contraceptive, before being discontinued due to severe side effects in patients after alcohol consumption (Heller et al. 1961, Zaneveld \& Waller 1989). However, when administered to male rats, WIN 18,446 caused a reduction in sperm concentration and motility without affecting Leydig cells (Brooks \& van der Horst 2003), and a study in male rabbits confirmed this was likely due to reduced aldehyde dehydrogenase $1 \mathrm{a} 2$ activity and retinoic acid production (Amory et al. 2011). Although gene expression studies on WIN 18,446-cultured mouse gonads demonstrated decreased Stra8 transcript levels after 48 h (Hogarth et al. 2011), no studies have investigated the impact of this inhibition on germ cell maturation and meiotic progression in the fetal ovary. Therefore, in this study, WIN 18,446 was used to explore the fate of germ cells after inhibition of retinoic acid synthesis and thus the relationship between retinoic acid, meiotic progression and primordial follicle assembly. Using a mouse fetal ovary culture model, where ovaries are dissected at e13.5 once meiosis has been initiated, we have shown that inhibition of retinoic acid synthesis with WIN 18,446 resulted in an acceleration of progression through prophase I and a subsequent increase in primordial follicle growth activation, thus suggesting a potential link between meiotic progression and primordial follicle formation and growth initiation.

\section{Materials and methods}

\section{Animals}

Experiments involving mice were approved by the University of Edinburgh Animal Research Ethics Committee and performed according to the UK Animal (Scientific Procedures) Act 1986. WT CD-1 mice were maintained and bred in an environmentally controlled room on a $12 \mathrm{~h}$ light: $12 \mathrm{~h}$ darkness photoperiod from $0700 \mathrm{~h}$ each day and fed ad libitum according to the UK Home Office and local University of Edinburgh ethical standards. To obtain fetuses for ovary culture experiments, mouse breeding harems were set up and females checked for the presences of a copulation plug; this was designated as embryonic day 0.5 (e0.5).

\section{Fetal ovary culture}

Pregnant timed-mated females were obtained at e13.5 and culled by cervical dislocation. Fetal ovaries with attached mesonephros were dissected from female embryos; the day of dissection was designated as day 0 of culture. Ovaries with attached mesonephros were cultured for either 2, 3 or 12 days on a $2 \%$ agar block in a $35 \mathrm{~mm}$ petri dish, incubated at $37^{\circ} \mathrm{C}$, $5 \% \mathrm{CO}_{2}$. For the first 3 days of culture (days 0-3), culture media contained Dulbecco's Minimal Essential medium (Fisher Scientific, UK) supplemented with $10 \%$ fetal bovine serum, $2 \mathrm{mM}$ L-glutamine, $10 \mu \mathrm{M} \beta$-mercaptoethanol and $1 \%$ sodium pyruvate. For subsequent days of culture, media were replaced with a simple culture medium consisting of $\alpha$-MEM (Fisher Scientific) supplemented solely with $3 \mathrm{mg} / \mathrm{mL}$ BSA. Medium was replaced with fresh medium every $72 \mathrm{~h}$. To inhibit retinoic acid production, fetal ovaries were cultured with doses of WIN 18,446 (CAS 1477-57-2, Tocris Bioscience) ranging from 0.01 $\mu \mathrm{M}$ to $5 \mu \mathrm{M}$ or equivalent concentration of DMSO solvent for the first 3 days of culture; medium was replaced on day 3 of culture thus removing the drug. For rescue experiments, $0.7 \mu \mathrm{M}$ of retinoic acid (Merck) was added to culture media in combination with WIN 18,446.

\section{Newborn ovary culture}

Ovaries were dissected from postnatal day 0 (P0) mice and cultured on floating polycarbonate membranes (Whatman, Sigma-Aldrich) in 24-well plates containing $\alpha$-MEM (Fisher Scientific) supplemented with $3 \mathrm{mg} / \mathrm{mL}$ BSA, incubated at $37^{\circ} \mathrm{C}, 5 \% \mathrm{CO}_{2}$. To investigate an independent effect on follicle activation, ovaries were cultured with $1 \mu \mathrm{M}$ of WIN 18,446 for 3 days. Subsequently, ovaries were transferred to drug- 
free medium, which was replaced every 48 h. Ovaries were cultured for a total of 6 days.

\section{RNA extraction, cDNA synthesis and RT-qPCR}

To assess the expression of retinoic acid response genes, cultured mouse fetal ovaries were collected in TRIzol (Fisher Scientific), homogenised using a motorised pellet pestle, and RNA was extracted using the RNeasy mini kit (Qiagen) according to manufacturer's instructions. RNA was reverse transcribed to cDNA using concentrated random primers and Superscript III reverse transcriptase (Fisher Scientific) according to manufacturer's instructions, and the cDNA synthesis reaction was diluted appropriately before proceeding. Primers for quantitative RT-PCR (RT-qPCR) were designed to amplify all transcript variants and are exon-spanning. Primer pair efficiencies were calculated with the LinReg PCR applet (Ramakers et al. 2003). Each reaction was performed in a final volume of $10 \mu \mathrm{L}$, with $1 \times$ Brilliant III SYBR Green qPCR Master Mix (Agilent), 20 pmol of each primer and $2 \mu \mathrm{L}$ of diluted cDNA. Primer sequences are as follows written in the 5' to 3' direction: Stra8 F GTTTCCTGCGTGTTCCACAAG, Stra8 R CACCCGAGGCTCAAGCTTC, $\operatorname{Rar} \beta \mathrm{F}$ TGT TTACCTGTTCACAAGCCA, $\operatorname{Rar} \beta$ R GGAGGAGACCGG AACAAGTT, Dazl F tggaccgaagcatacagaca, Dazl $\mathrm{R}$ actgcccgacttcttctgaa, Syсp3 F cagagccagagaatgaaagca, Sуср3 $\mathrm{R}$ attgccatctcttgctgct, and see van den Bergen et al. 2009 for Mapk1 (Mitogen-activated protein kinase 1) and Canx (Calnexin precursor) sequences. Each cDNA sample was analysed in triplicate. Target genes were normalised to the geometric mean expression of Canx and Mapk1 (van den Bergen et al. 2009). Data analysis for relative quantification of gene expression and calculation of S.D. was performed as outlined (Livak \& Schmittgen 2001, Vandesompele et al. 2002).

\section{Immunohistochemistry}

Single immunostaining for Tra98 (germ cell specific antigen) was used to identify germ cells for counting after 3 days of culture with WIN 18,446. Double immunostaining for Msy2 and Tra98 was used to assess oocyte maturation on day 12 of culture (ovaries only exposed to WIN 18,446 for first 3 days of culture). Cultured fetal ovaries were fixed for $2 \mathrm{~h}$ in Bouins solution. After processing, tissue was sectioned at $5 \mu \mathrm{m}$, dewaxed, rehydrated and antigen retrieval carried out in $0.01 \mathrm{M}$ citrate buffer $\mathrm{pH}$ 6. Endogenous peroxidase activity was blocked with Dako REAL Peroxidase-Blocking solution (Agilent). Tissues were blocked in PBS containing $5 \%$ BSA and $20 \%$ normal goat serum and then incubated overnight at $4^{\circ} \mathrm{C}$ with primary antibodies diluted in blocking serum (Tra98: 1 in 200 (Ab82527, Abcam), Msy2: 1 in 400 (Ab82527, Abcam)). Single immunostaining for Tra98 was carried out using ImmPRESS reagent (Vector Laboratories, UK) according to manufacturer's instructions, and slides were counterstained with hematoxylin before mounting. For double immunofluorescence, tissues were incubated with a peroxidase-conjugated antibody for $30 \mathrm{~min}$ at room temperature, before visualisation using Tyramide Signal Amplification (TSA) (PerkinElmer) according to manufacturer's instruction. The first primary antibody (Msy2) was then removed by a second antigen retrieval performed by microwaving slides in boiling $0.01 \mathrm{M}$ citrate buffer for $2.5 \mathrm{~min}$. After cooling, slides were blocked as before and incubated overnight at $4^{\circ} \mathrm{C}$ with anti-rat Tra98 ( 1 in 400). Secondary antibody and visualisation steps were carried out as mentioned previously, before counterstaining with DAPI and mounting. Images were captured using an Axioscan slide scanner (Carl Zeiss) and a 710 LSM confocal microscope (Carl Zeiss) with Zen 2009 software.

\section{Chromosome spreads and immunostaining}

Ovaries were taken at day 2 of culture (that is $48 \mathrm{~h}$ post culture setup), and for each $n$, five ovaries were pooled to ensure enough oocytes for quantification and oocyte chromosome spreads were prepared as described previously (Peters et al. 1997). For immunostaining, slides were first washed in PBS, then blocked in PBS containing 0.15\% BSA, 0.1\% Tween 20 and 5\% goat serum. Slides were then incubated with mouse anti-Sycp3 (1:500. Abcam, ab97672), rabbit anti-Rpa (1:300, He et al. 1995) and guinea pig anti-Sycp1 (1:200, Bolcun-Filas et al. 2009) diluted in block buffer. Alexa Fluorconjugated secondary antibodies (Invitrogen) were used at a 1:500 dilution, and $2 \mathrm{ng} / \mu \mathrm{L}$ DAPI was used to fluorescently stain DNA before mounting. Images were captured using a Zeiss Imager Z1 fluorescence microscope with Plan-neofluar objectives (Carl Zeiss), and image capture was performed using Zen 2009 software.

\section{Meiotic staging}

Oocytes were staged, blinded to treatment, as outlined in Crichton et al. 2017, 2018. Briefly, staging was based on synaptonemal complex formation/dissolution, using staining patterns of the axial element protein Sycp3, which marks the axis of each homologue, and the transverse filament protein Sycp1, which marks regions of chromosome synapsis. All Sycp3-positive oocytes were scored. Leptotene nuclei contained fragmented axial elements and a lack of synapsis indicated by the absence of co-localisation between Sycp3 and the transverse filament marker Sycp1. Zygotene nuclei were identified by extensive axial element formation and partial synapsis. Pachytene oocytes contained at least one fully synapsed pair of homologous chromosomes. Recombination foci marked by Rpa were used to distinguish between zygotene and diplotene, with Rpa foci being absent in diplotene nuclei (Shi et al. 2019); no diplotene nuclei were observed.

\section{Germ cell counts and histological follicle assessment}

Fetal (e13.5) ovaries were fixed for $2 \mathrm{~h}$ in Bouins solution on days 3 and 12 of culture, and newborn (p0) ovaries were fixed for $2 \mathrm{~h}$ in Bouins solution on day 6 of culture, paraffin wax-embedded, sectioned and stained for Tra98 (germ cell marker) or with hematoxylin and eosin. Assessment of germ cell number was carried out on $5-\mu \mathrm{M}$ thick tissue sections and every sixth section, spanning the entire volume of the ovary (6-10 sections, depending on tissue orientation), with the 
Abercrombie correction factor being applied to raw counts to estimate total germ cell number per ovary (Abercrombie 1946). Assessment of follicle stage and health was carried out (blinded) on every sixth section of day 12 of e13.5 cultured ovaries or day 6 of P0 cultured ovaries. A follicle was counted only where the analysed section contained an oocyte with a visible germinal vesicle. Primordial follicles (PMF) were defined as having only squamous pre-granulosa cells, transitionary follicles (TRNS) had both squamous and cuboidal granulosa cells, and primary follicles (PRIM) had a complete layer of cuboidal granulosa cells. Follicle health was assessed as described previously (Stefansdottir et al. 2016).

\section{Statistical analysis}

All data are shown as mean \pm S.E.M. and were analysed using GraphPad Prism 7 software (GraphPad Software Inc.). MannWhitney $U$-test and Kruskal-Wallis test statistics were carried out as appropriate. A $P$ value of $<0.05$ was considered statistically significant.

\section{Results}

\section{WIN 18,446 inhibits the expression of retinoic acid} response genes Stra8 and Rarb in mouse fetal ovaries

To examine the effect of WIN 18,446 on the fetal ovary, ovaries were isolated from e13.5 mice and cultured with doses of WIN 18,446 ranging from $0.01 \mu \mathrm{M}$ to $5 \mu \mathrm{M}$ or vehicle only. RT-qPCR was used to evaluate the expression of retinoic acid response genes Stra8 and Rarb on day 2 of culture (Fig. 1). Culture with the lowest dose of WIN 18,446 $(0.01 \mu \mathrm{M})$ resulted in approximately $50 \%$ reduction in the transcript levels of Stra8 $(P=0.008)$, and a clear dose response was observed with the $5 \mu \mathrm{M}$ dose, causing approximately $97 \%$ reduction in Stra8 expression (Fig. 1A). To demonstrate that this reduction was caused by reduced retinoic acid synthesis, fetal ovaries were cultured with $0.7 \mu \mathrm{M}$ of retinoic acid in combination with $2 \mu \mathrm{M}$ of WIN 18,446; this stimulated Stra8 expression at least 20-fold when compared to ovaries cultured with 2 $\mu \mathrm{M}$ of WIN 18,446 alone $(P=0.016)$. Furthermore, the addition of retinoic acid increased Stra8 expression 0.5 fold above what was observed in vehicle-cultured ovaries $(P=0.014)$. Rarb expression was also reduced by WIN 18,446 with a similar dose response (Fig. 1B). The addition of retinoic acid increased Rarb transcript levels compared to ovaries cultured with $2 \mu \mathrm{M}$ of WIN 18,446 only $(P=0.008)$, to a similar level of expression in vehicle-cultured ovaries (Fig. 1B). However, culture with WIN 18,446 did not have any significant impact on the expression of Sycp3 and Dazl in e13.5 cultured ovaries (Fig. 1C and D); both genes are important for meiotic initiation and progression but are not considered to be direct targets of retinoic acid. These gene expression data indicate that treatment with WIN 18,446 is sufficient to prevent retinoic acid synthesis by
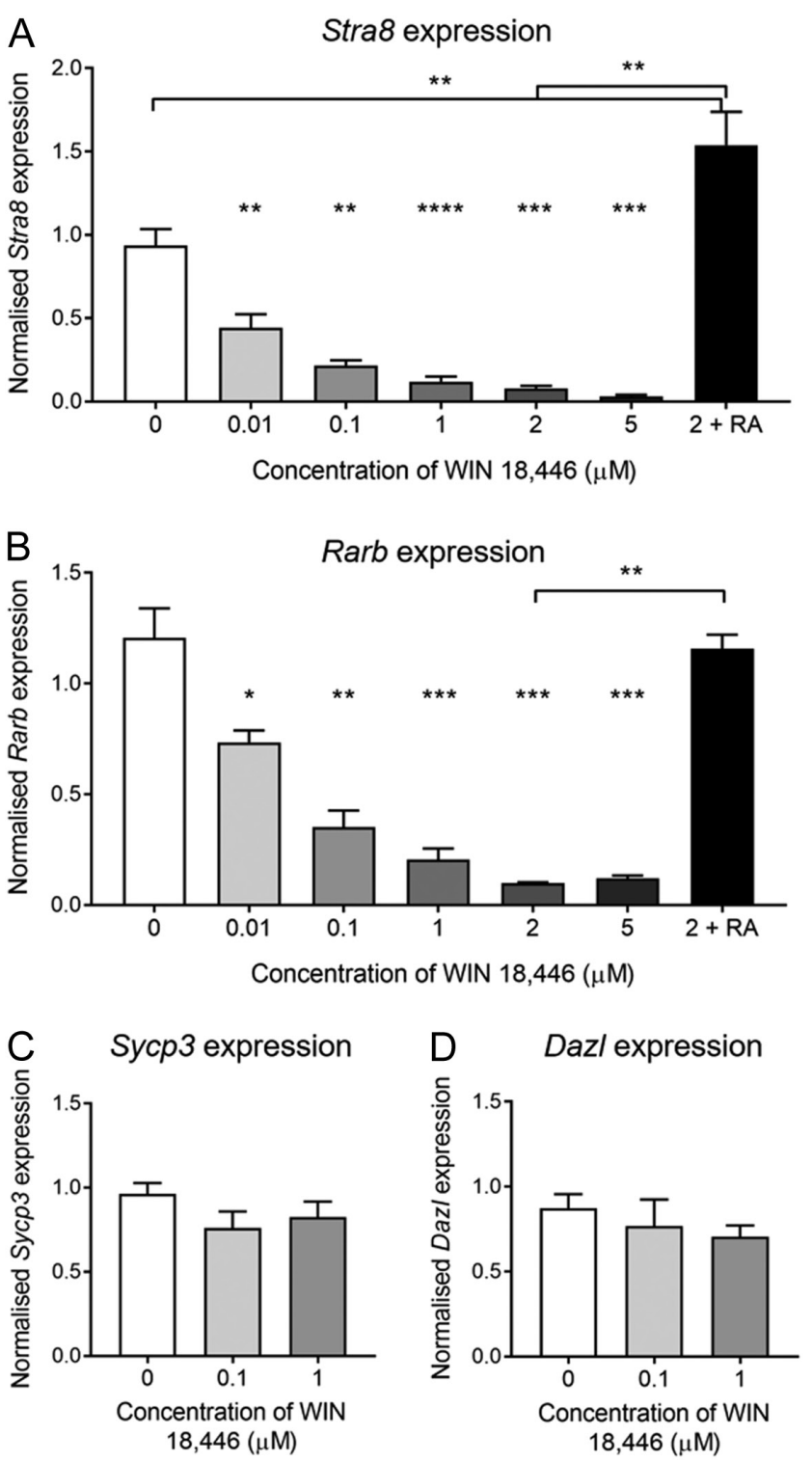

Figure 1 Retinoic acid response gene expression in e13.5 mouse ovaries cultured for 2 days with WIN 18,446 or vehicle control. (A) Stra8, (B) Rark, (C) Sycp3 and (D) Dazl mRNA expression in ovaries cultured for 2 days with WIN 18,446 doses ranging from $0.01 \mu \mathrm{M}$ to $5 \mu \mathrm{M}, 2 \mu \mathrm{M}$ WIN 18,446 with $0.7 \mu \mathrm{M}$ of retinoic acid, or vehicle control. Graph displays mean of $n=5$ ovaries for each group. Error bars indicate \pm S.E.M. ${ }^{*} P<0.016$; ${ }^{* *} P<0.008$; $* * * P<0.0007$.

blocking the conversion of retinol to retinoic acid in the mouse fetal ovary, as previously demonstrated.

\section{WIN 18,446 causes germ cell loss at $2 \mu \mathrm{M}$ but not at $0.1 \mu \mathrm{M}$ and $1 \mu \mathrm{M}$}

Given the dramatic changes in Stra8 and Rarb expression following culture with WIN 18,446, we sought to identify whether, in part, this may have been caused by a reduction in germ cell number. Immunostaining for the germ cell marker Tra98 was utilised to facilitate germ cell 
quantification after 3 days of culture with WIN 18,446 (Fig. 2A), and we chose to investigate the three midrange doses $0.1,1$ and $2 \mu \mathrm{M}$, as these resulted in $>50 \%$ reduction in the expression of Stra8 and Rarb. There was no significant difference in total germ cell number per ovary between ovaries cultured with WIN 18,446 at 0.1 or $1 \mu \mathrm{M}$ and vehicle controls (Fig. 2B). Therefore, this finding confirms that the significant reduction in Stra8 and Rarb transcript levels at these lower doses depicted in Fig. 1 is due to the action of WIN 18,446 inhibiting retinoic acid synthesis rather than decreased germ cell number. Treatment with $2 \mu \mathrm{M}$ WIN 18,446, however, resulted in a reduction in germ cell number by approximately $40 \%$; this loss was prevented by the addition of $0.7 \mu \mathrm{M}$ retinoic acid (Fig. 2C), suggesting that retinoic acid may have a role in germ cell survival.

\section{Reduced retinoic acid synthesis accelerates oocyte progression through meiotic prophase I in cultured mouse fetal ovaries}

Double immunostaining for Tra98 and Msy2 as differential molecular markers of meiotic progression was carried out to ascertain the effect of WIN 18,446 on oocyte progression through prophase I. This experiment was carried out using only the $0.1 \mu \mathrm{M}$ and $1 \mu \mathrm{M}$ doses of WIN 18,446, given that these doses caused $>50 \%$ reduction in the expression of Stra8 and Rarb without any germ cell loss. Immunostaining was performed on ovaries cultured for 12 days, a time-frame which spans the onset of meiosis in germ cells to the formation of primordial follicles and the subsequent initiation of growth in some follicles. Tra98 is expressed in the nucleus of germ cells from their arrival at the gonadal ridge up until arrest at dictyate, when oocytes acquire a layer of granulosa cells (Enders \& May 1994), while Msy2 expression in the oocyte cytoplasm correlates with arrival at the diplotene stage (though it is not a marker for diplotene arrest per se) (Burks et al. 2019) (Fig. 3A). Given the proposed importance of retinoic acid in initiating meiosis, we hypothesised that culture with $1 \mu \mathrm{M}$ of WIN 18,446 would impede meiotic progression; however, we observed that there were more Tra98 positive oocytes in vehicle-control-cultured ovaries compared with WIN 18,446-cultured ovaries (Fig. 3B and C). Quantification of oocytes as Tra98 positive (including those that co-expressed Msy2) or Tra98 negative showed that $14.2 \%( \pm 3.6 \%)$ of oocytes expressed Tra98 in vehicle cultured-ovaries, while only $3.2 \%( \pm 1.7 \%)$ of oocytes were Tra98 positive in ovaries cultured with $1 \mu \mathrm{M}$ of WIN $18,446(P<0.0001)$ (Fig. $3 \mathrm{E})$. Culture with $1 \mu \mathrm{M}$ of WIN 18,446 and $0.7 \mu \mathrm{M}$ of retinoic acid rescued this phenotype, with the proportion of Tra98 positive oocytes $(14 \pm 2.1 \%)$ returning to what was observed in vehicle-cultured ovaries $(P<0.0001$ vs WIN 18,446 only) (Fig. 3D and E). Again, a clear dose response was observed, as ovaries cultured with $0.1 \mu \mathrm{M}$ of WIN 18,446 had proportions of Tra98-positive and Msy2-positive oocytes that were in between vehiclecultured ovaries and $1 \mu \mathrm{M}$ WIN 18,446-cultured ovaries (data not shown). With regards to location of these oocytes, there appeared to be no obvious pattern in distribution of Tra98-positive and Msy2-positive within vehicle-control-cultured ovaries; however, oocytes positive for Tra98 only and both Tra98 and Msy2 were more frequently located on the periphery of WIN 18,446-cultured ovaries (highlighted by arrows in Fig. 3C). Taken together, these data suggest that culture with WIN 18,446 actually accelerates progression through prophase I, as ovaries cultured with WIN 18,446 had significantly fewer Tra98 positive oocytes than vehicle cultured-ovaries.

As the Tra98/Msy2 quantification data was collected at the end of the culture period, we investigated whether this accelerated progression could be observed at an earlier point in culture. Oocyte chromosome spreads were therefore prepared from ovaries on day 2 of culture to capture oocytes in early meiotic prophase I. For sub-staging, oocytes were stained with antibodies against components of the synaptonemal complex: axial
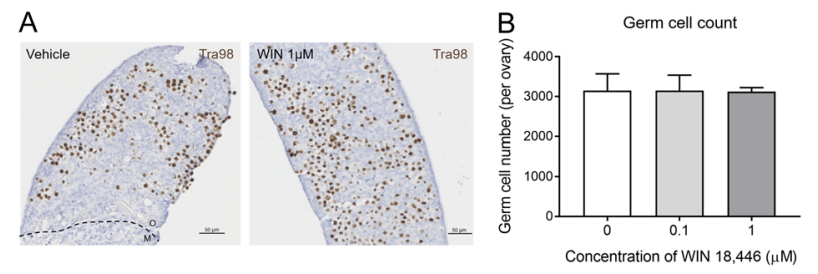

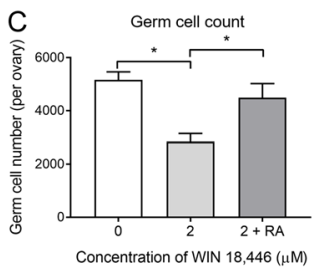

Figure 2 Germ cell number in e13.5 mouse ovaries cultured for 3 days with WIN 18,446 or vehicle control. (A) Representative image of Tra98 immunostaining used to quantify germ cells in vehicle-control and WIN 18,446 ovaries cultured for 3 days. Dashed line denotes border between mesonephros $(\mathrm{M})$ and ovary $(\mathrm{O})$. Scale bar $=50 \mu \mathrm{M}$. (B) Germ cell number was quantified after 3 days of culture with $0.1 \mu \mathrm{M}$ and $1 \mu \mathrm{M}$ of WIN 18,446 or vehicle control. Error bars indicate \pm S.E.M. $n=5$ ovaries per group. (C) Germ cell number was quantified after 3 days of culture with $2 \mu \mathrm{M}$ WIN 18,446, $2 \mu \mathrm{M}$ WIN 18,446+0.7 $\mu \mathrm{M}$ retinoic acid or vehicle control. Error bars indicate \pm S.E.M. $n=5$ ovaries per group. ${ }^{*} P=0.029$. 


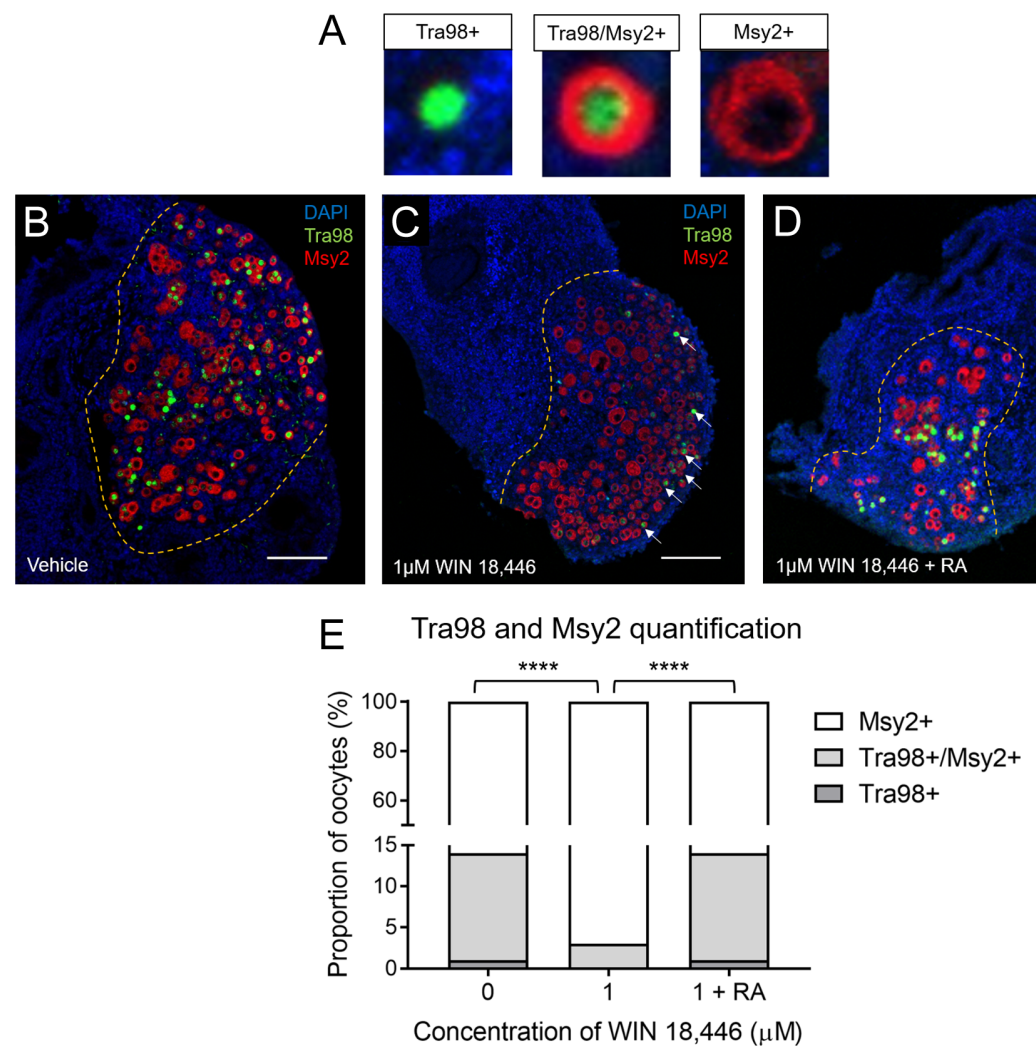

Figure 3 Tra98 and Msy2 double immunostaining on e13.5 mouse ovaries cultured for 12 days with WIN 18,446 or vehicle control. (A, B, C and D) Representative images of Tra98 (green) and Msy2 (red) immunostaining used to quantify germ cells in ovaries treated with vehicle-control, $1 \mu \mathrm{M}$ WIN 18,446, and $1 \mu \mathrm{M}$ WIN 18,446+0.7 $\mu \mathrm{M}$ RA and cultured for 12 days. Yellow dashed line denotes border between mesonephros and ovary. Scale bar $=100 \mu \mathrm{M}$. (E) Quantification of Tra98 and Msy2 immunostaining in vehicle-control, $1 \mu \mathrm{M}$ WIN 18,446 and $1 \mu \mathrm{M}$ WIN 18,446+0.7 $\mu \mathrm{M}$ retinoic acid ovaries cultured for 12 days. Error bars indicate \pm S.E.M. $n=5$ ovaries per group. ${ }^{* * * *} P<0.0001$.

element protein Sycp3, and transverse filament protein Sycp1, as well as the recombination protein Rpa. A total of 258 oocytes from vehicle cultured-ovaries and 250 from ovaries cultured with $1 \mu \mathrm{M}$ WIN 18,446 were analysed, blinded to the treatment group, using this dose only to maximise likely effects. Oocytes at three different stages were identified: leptotene, with short fragments of axial element but no synapsis; zygotene, with nuclei containing some regions of axial element undergoing synapsis, but this was not complete along the axial length; and pachytene, which had at least one pair of fully synapsed chromosomes. No diplotene oocytes were observed. Rpa recombination foci were present at all stages identified, but were less prevalent in pachytene oocytes (Fig. 4A).

In vehicle-cultured ovaries, an average of $17.6 \%$ $( \pm 2.9)$ of oocytes were in leptotene, $38.7 \%( \pm 1.7)$ in zygotene, and $43.7 \%( \pm 1.5)$ in pachytene (Fig. $4 B)$. While there was no significant difference in the proportion of leptotene oocytes, representation of the different substages in ovaries cultured with WIN 18,446 showed a slight skew toward the latter stages of prophase I, with a significant decrease in the proportion of zygotene oocytes compared to vehicle-cultured ovaries $(P=0.029)$ and a significant increase in the proportion of pachytene oocytes $(P=0.029)$. In WIN 18,446 -cultured ovaries, on average $25.0 \%( \pm 4.7)$ of oocytes were in leptotene, $21.9 \%( \pm 2.6)$ in zygotene, and $53.2 \%( \pm 3.5)$ in pachytene (Fig. 4B). These data demonstrate that the acceleration of meiosis seen on day 12 of culture caused by reduced retinoic acid synthesis as a result of culture with WIN 18,446 can be observed as early as $48 \mathrm{~h}$ after treatment.

\section{Reduced retinoic acid synthesis accelerates follicle activation in culture mouse fetal ovaries}

As culture with WIN 18,446 accelerated oocyte progression through prophase I, we explored the impact of this acceleration on primordial follicle formation and subsequent follicle growth initiation, using $0.1 \mu \mathrm{M}$ and 1 $\mu \mathrm{M}$ doses of WIN 18,446, as these had no effect on germ cell number in earlier experiments. At day 12 of culture, the total number of follicles present in vehicle and WIN 18,446-cultured ovaries was assessed and follicles were staged as primordial, transitionary or primary. There was no significant difference in the total follicle number between vehicle-cultured ovaries and ovaries cultured with either $0.1 \mu \mathrm{M}$ or $1 \mu \mathrm{M}$ of WIN 18,446 (Fig. 5A and B). There was also no difference in follicle health post culture with WIN 18,446, as approximately $4 \%$ of all follicles in each treatment group were unhealthy. However, ovaries cultured with both doses of WIN 18,446 had proportionally fewer primordial follicles and more transitionary follicles compared with vehicle-cultured ovaries $(P=0.032$ and $P=0.040$, respectively) (Fig. 5C and D). Ovaries cultured with WIN 18,446 also had fewer oocytes within germ cell nest structures, suggesting these structures had broken down to form primordial follicles earlier than germ cell 

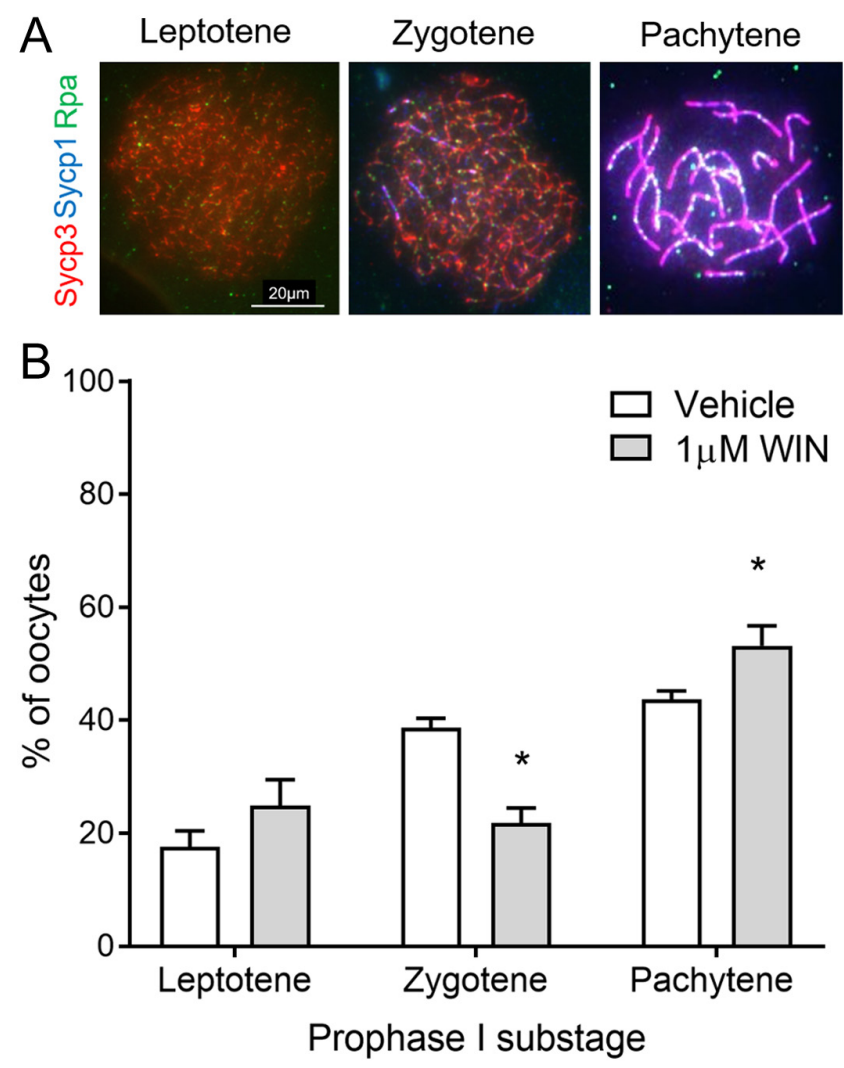

Figure 4 Sub-staging meiotic prophase I oocytes from e13.5 mouse ovaries cultured for 2 days with WIN 18,446 or vehicle control. (A) Immunostaining of Sycp3 (red), Sycp1 (blue) and Rpa (green) to assess axial element and transverse filament formation, respectively. Representative merged images displayed. Scale bar $=20 \mu \mathrm{M}$. (B) Relative proportions of prophase I substages from ovaries cultured for 2 days with vehicle-control and $1 \mu \mathrm{M}$ WIN 18,446. Graph displays mean percentages from four experiment repeats, and a total of 258 oocytes from vehicle cultured-ovaries and 250 from WIN 18,446 cultured ovaries were analysed. Error bars indicate \pm S.E.M. $* P<0.04$.

nests in vehicle-cultured ovaries, although this was only significant with $1 \mu \mathrm{M}$ of WIN $18,446(P=0.05 ; 0.01$ $\mu \mathrm{M}$ dose $P=0.06$ ) (Fig. 5E). As the evidence of follicle activation was greatest using $1 \mu \mathrm{M}$ of WIN 18,446, we sought to investigate whether this dose would have a similar effect on follicle growth in P0 ovaries, that is, independently of an effect on meiotic progression. There was no indication of increased follicle growth when P0 ovaries were cultured with $1 \mu \mathrm{M}$ of WIN 18,446 (Fig. 5F). Thus, these data suggest that the advanced completion of meiotic prophase in oocytes after treatment with WIN 18,446 results in an increased rate of initial follicle growth activation, with more primordial follicles in WIN 18,446 treated ovaries initiating growth than in their control counterparts.

\section{Discussion}

This present study, to our knowledge, is the first in-depth investigation into the impact of inhibition of retinoic acid synthesis on meiotic progression in the fetal ovary and its relationship with subsequent follicle formation and growth initiation. We have used the compound WIN 18,446 to inhibit retinoic acid synthesis in a fetal mouse ovary culture system that supports germ cell survival and progression through prophase I of meiosis up to diplotene arrest, followed by primordial follicle formation and subsequent growth initiation (Stefansdottir et al. 2016, Rosario et al. 2019). Fetal ovaries are cultured from e13.5, a stage when germ cells are committed to oocyte development and enter the first stages of meiosis (Adams \& McLaren 2002); thus, in this study we are investigating the role of retinoic acid on meiotic progression, distinct from its initiation. Previous studies have demonstrated that treatment with WIN 18,446 results in a vitamin A-deficiency phenotype in the testes of adult male rabbits (Amory et al. 2011), and experiments in adult male mice (Brooks \& van der Horst 2003) and in cultured testes from adult, neonatal and embryonic mice have shown a reduction in the levels of tissue retinoic acid and Stra8 transcripts with treatment with WIN 18,446 (Hogarth et al. 2011). However, there has been little research into the effects of this compound on the ovary and female fertility, with only one study reporting decreased Stra8 expression in e11.5 mouse ovaries cultured for $48 \mathrm{~h}$ with $1 \mu \mathrm{M}$ WIN 18,446 (Hogarth et al. 2011). We have confirmed and furthered this finding by demonstrating that WIN 18,446 concentrations as low as $0.01 \mu \mathrm{M}$ are sufficient to cause at least $50 \%$ reduction in Stra8 and Rarb expression in e13.5 mouse fetal ovaries. Importantly, we have also demonstrated this altered gene expression is not due to germ cell loss, not investigated in the previous study. While WIN 18,446 doses up to $1 \mu \mathrm{M}$ were not damaging, the germ cell loss evident at higher doses of WIN 18,446 could be rescued with the addition of retinoic acid, highlighting the potential of retinoic acid as a germ cell survival factor; however, this notion needs further exploration.

A growing body of evidence implicates retinoic acid, with the subsequent transcription of Stra8, as a key requirement to drive the initiation of meiosis in both male and female mammals. This has resulted in a mechanistic model involving the interplay of retinoic acid, enzymes involved in its synthesis and the receptors to which it binds, and in the prepubertal testis, protection against its actions by metabolising enzymes (Feng et al. 2014). It is important to note that much of this work does not detail the involvement of retinoic acid once meiosis is underway. However, conversely to expectations based on this model, after retinoic acid inhibition in our culture system, germ cells appear to have progressed through stages of prophase I faster than germ cells in vehiclecontrol-cultured ovaries. This acceleration was evident as early as $48 \mathrm{~h}$ in culture, with chromosome spread data from vehicle-control-cultured ovaries having a lower proportion of zygotene oocytes (39\% vs $22 \%$ after 

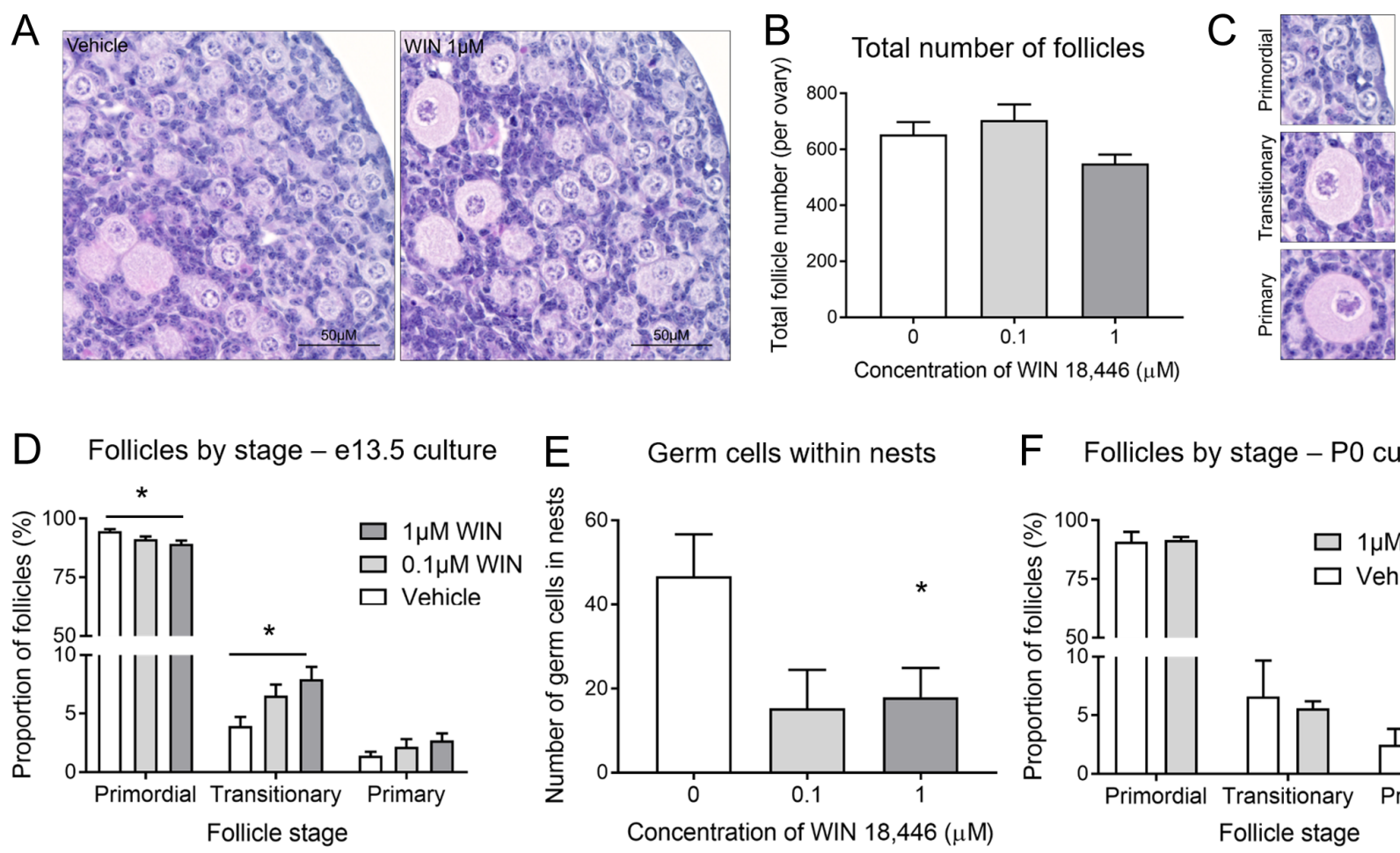

F Follicles by stage - P0 culture

Figure 5 Assessment of follicles from e13.5 mouse ovaries cultured for 12 days with WIN 18,446 or vehicle control (A) Representative hematoxylin and eosin images from vehicle-control and $1 \mu \mathrm{M}$ WIN 18,446 e13.5 ovaries cultured for 12 days. Scale bars $=50 \mu \mathrm{M}$. (B) Graph depicts total number of follicles in vehicle-control and WIN 18,446 e13.5 ovaries cultured for 12 days. Error bars indicate \pm S.E.M. $n=6$ ovaries per group. (C) Representative hematoxylin and eosin images of primordial (PMF), transitionary (TRNS) and primary (PRIM) follicles. Primordial follicles (PMF) were defined as having only squamous pre-granulosa cells, transitionary follicles (TRNS) were considered to have both squamous and cuboidal granulosa cells, and primary follicles (PRIM) had a complete layer of cuboidal granulosa cells. (D) Graph depicts the proportion of follicles staged as PMF, TRNS or PRIM in vehicle-control and WIN 18,446 e13.5 ovaries cultured for 12 days. Error bars indicate \pm S.E.M. $n=6$ ovaries per group. $P<0.001$. (E) Graph depicts number of germ cells found in nest structures in vehicle-control and WIN 18,446 e13.5 ovaries cultured for 12 days. All germ cells found in nests in each ovary were counted. Error bars indicate \pm S.E.M. $n=6$ ovaries per group, $P=0.05$ (F) Graph depicts the proportion of follicles staged as PMF, TRNS or PRIM in vehicle-control and $1 \mu \mathrm{M}$ WIN 18,446 P0 ovaries cultured for 6 days. Error bars indicate \pm S.E.M. $n=4$ ovaries per group.

WIN 18,446 treatment), while WIN 18,446-cultured ovaries had many oocytes in pachytene (53\% vs $44 \%$ in vehicle controls). This result is in contrast to findings in vitamin A deficient rat embryonic ovaries, where, at e18.5, vitamin A deficiency had no impact on germ cell number but Stra8 induction was absent and most germ cells were unable to enter meiosis, as assessed by a lack of Sycp3 staining usually found at the onset of leptotene (Li \& Clagett-Dame 2009). Furthermore, although germ cells should have entered meiosis by e18.5, the expression of the pluripotency marker Oct4 persisted; the commitment of ovarian germ cells to meiosis is normally associated with the downregulation of Oct4 (Pesce et al. 1998). Vitamin A deprivation in male rodents also results in spermatogenic arrest (Morales \& Griswold 1987, Griswold et al. 1989, van Pelt \& de Rooij 1991, Li et al. 2011); however, large doses of retinoic acid can induce resumption of meiosis in male and female vitamin A deficiency models, suggesting that it is retinoic acid and not its precursor retinol that is the active factor (van Pelt \& de Rooij 1991, Li \& Clagett-Dame 2009).
Given that these data are from models where retinoic acid levels were reduced from conception, it would appear that timing of retinoic acid inhibition may be key in determining the subsequent effects on meiotic onset and progression; as germ cells have already initiated meiosis in our e13.5 culture model, we are able to identify potential effects on meiotic progression/germ cell development that are distinct from the need for retinoic acid for initiation of meiosis. Indeed, culture of mouse fetal ovaries at e11.5 with the synthetic retinoic acid receptor antagonist BMS has the expected effect of blocking meiosis initiation (Minkina et al. 2017).

Accelerated progression through various stages of meiosis I and II has been previously reported. Specifically related to prophase I, deletion of the kinase Kin-18 or checkpoint protein Pch-2 in C. elegans leads to accelerated prophase I progression, aberrant oocyte development, and ectopic apoptosis (Deshong et al. 2014, Yin et al. 2016). In chickens, exposure to bisphenol A, an endocrine-disrupting chemical with weak oestrogenic activity, has been reported to affect 
the formation of primordial follicles by promoting meiotic progression of oocytes via estrogen receptor beta signaling pathways (Yu et al. 2018). However, the most relevant phenotype is that of Sycp1 depletion in e21 rat fetal ovaries, which resulted in a decrease and increase in Tra98 and Mys2 expressing oocytes, respectively (Paredes et al. 2005). While those authors concluded that expression of Msy2 reflected attainment of diplotene, it has recently been demonstrated that it is not an exact marker of diplotene per se as demonstrated via chromosome spreads, although it does correlate with arrival at the diplotene stage (Burks et al. 2019). Nonetheless, using this Tra98/Msy2 immunostaining strategy we too observed a reduced proportion of Tra98 positive oocytes in ovaries where retinoic acid synthesis was blocked by WIN 18,446, and this effect was rescued with the addition of retinoic acid. As Tra98 is found in the nucleus of germ cells prior to dictyate arrest (it is lost from spermatocytes in late pachytene and diplotene and thought to be lost from oocytes at the same time (Enders \& May 1994)), it would appear that the reduction of retinoic acid caused an acceleration of germ cell maturation.

In addition to accelerated prophase progression, Paredes et al. also observed increased folliculogenesis in Sycp1-deficient ovaries, with primordial follicles assembled earlier and in greater numbers than in control ovaries and more primary follicles indicating increased initiation of follicle growth (Paredes et al. 2005). Although we did not observe any difference in total follicle number after retinoic acid inhibition, we did see evidence of earlier oocyte nest breakdown and a modest increase in follicle activation in WIN 18,446-cultured ovaries; that is, these ovaries had proportionally fewer primordial and more transitionary follicles compared with vehicle-control-cultured ovaries. Interestingly, when we cultured newborn P0 ovaries with WIN 18,446 for the same duration (3 days exposure), we did not observe any increase in follicle growth. This is unlike the effect of growth factors such as FGF (Nilsson \& Skinner 2004) and PDGF (Nilsson et al. 2006), which have been shown to increase levels of Kit-ligand (Parrott \& Skinner 1999) and enhance the transition of primordial follicles to primary stage in newborn rat ovaries. Therefore, this would indicate that the alterations here identified in follicle activation after culture with WIN 18,446 at e13.5 are related to events taking place within germ cells during the first 3 days of culture. Furthermore, as WIN 18,446 is removed from the culture medium after this, it would seem these early changes in germ cells not only effect meiotic progression but also create knock-on effects for primordial follicle formation and growth initiation. From our data, one may speculate that the effects on meiotic progression and follicle activation may even be linked, as suggested by the 'production line hypothesis'. According to this hypothesis, germ cells in female mammals become committed to meiosis and enter prophase sequentially in fetal life and the oocytes thus generated are activated to grow in the same sequence as that of meiotic entry (Polani \& Crolla 1991, Hirshfield 1992, Mork et al. 2012), although findings in human and mouse fetal oocytes suggest otherwise (Speed \& Chandley 1983, Rowsey et al. 2014). It is worth noting that our experiments do not exclude the impact of reduced retinoic acid synthesis on the surrounding somatic environment and the indirect effect this could have on germ cell maturation, given the bidirectional communication between these two cell types. Indeed, we have previously demonstrated the expression of retinoic acid receptors in pre-granulosa cells of the human fetal ovary (Childs et al. 2011). However, deletion of all three retinoic acid receptors in the fetal mouse ovary at the time of sex determination did not significantly affect ovarian differentiation, follicle development or female fertility, thus suggesting that retinoic acid signaling is dispensable for somatic development and function in the mouse ovary (Minkina et al. 2017).

In conclusion, the present data demonstrate that WIN 18,446 is highly effective at reducing retinoic acid synthesis in the mouse fetal ovary, enabling investigation into how retinoic acid regulates germ cell development after the initiation of meiosis in females. We have shown that reduced retinoic acid synthesis resulted in accelerated meiotic prophase I progression and increased follicle growth activation in mouse ovaries cultured at e13.5. These data thus suggest the importance of retinoic acid not just in the onset of meiosis but in germ cell survival and subsequent meiotic progression and support a link between meiosis and follicle formation.

\section{Declaration of interest}

The authors declare that there is no conflict of interest that could be perceived as prejudicing the impartiality of the research reported.

\section{Funding}

The authors' work in this field is supported by grants from the Medical Research Council (G1100357 to R A A, MR/N022556/1 to the MRC Centre for Reproductive Health) and the Biotechnology and Biological Sciences Research Council (BB/R015635/1 to R A A and R R).

\section{Author contribution statement}

R R and R A A designed the experiments. R R, H L S and E W carried out experiments. $\mathrm{R} R$ wrote the manuscript. All authors contributed to data interpretation, editing the manuscript, and its final approval. 


\section{Acknowledgements}

The authors are grateful to Dr lan Adams for discussion and the generous gift of antibodies and Dr James Crichton for his guidance in meiotic staging. The authors would also like to acknowledge the members of the SURF imaging suite at the University of Edinburgh, especially, Mike Millar for his assistance.

\section{References}

Abercrombie M 1946 Estimation of nuclear population from microtome sections. Anatomical Record 94 239-247. (https://doi.org/10.1002/ ar.1090940210)

Adams IR \& Mclaren A 2002 Sexually dimorphic development of mouse primordial germ cells: switching from oogenesis to spermatogenesis. Development 129 1155-1164.

Amory JK, Muller CH, Shimshoni JA, Isoherranen N, Paik J, Moreb JS, Amory Sr DW, Evanoff R, Goldstein AS \& Griswold MD 2011 Suppression of spermatogenesis by bisdichloroacetyldiamines is mediated by inhibition of testicular retinoic acid biosynthesis. Journal of Andrology 32 111-119. (https://doi.org/10.2164/jandrol.110.010751)

Baltus AE, Menke DB, Hu YC, Goodheart ML, Carpenter AE, De Rooij DG \& Page DC 2006 In germ cells of mouse embryonic ovaries, the decision to enter meiosis precedes premeiotic DNA replication. Nature Genetics 38 1430-1434. (https://doi.org/10.1038/ng1919)

Bendsen E, Byskov AG, Andersen CY \& Westergaard LG 2006 Number of germ cells and somatic cells in human fetal ovaries during the first weeks after sex differentiation. Human Reproduction 21 30-35. (https:// doi.org/10.1093/humrep/dei280)

Bolcun-Filas E, Hall E, Speed R, Taggart M, Grey C, De Massy B, Benavente R \& Cooke HJ 2009 Mutation of the mouse Syce1 gene disrupts synapsis and suggests a link between synaptonemal complex structural components and DNA repair. PLoS Genetics 5 e1000393. (https://doi.org/10.1371/journal.pgen.1000393)

Bowles J, Feng CW, Miles K, Ineson J, Spiller C \& Koopman P 2016 ALDH1A1 provides a source of meiosis-inducing retinoic acid in mouse fetal ovaries. Nature Communication 7 10845. (https://doi.org/10.1038/ ncomms10845)

Bowles J, Knight D, Smith C, Wilhelm D, Richman J, Mamiya S, Yashiro K, Chawengsaksophak K, Wilson MJ, Rossant J et al. 2006 Retinoid signaling determines germ cell fate in mice. Science 312 596-600. (https://doi.org/10.1126/science.1125691)

Brooks NL \& Van Der Horst G 2003 Short-term effects of N'Nbis(dichloroacetyl)-1,8-octamethylenediamine (WIN 18446) on thetestes, selected sperm parameters and fertility of male CBA mice. Laboratory Animals 37 363-373. (https://doi.org/10.1258/002367703103051921)

Burks DM, Mccoy MR, Dutta S, Mark-Kappeler CJ, Hoyer PB \& Pepling ME 2019 Molecular analysis of the effects of steroid hormones on mouse meiotic prophase I progression. Reproductive Biology and Endocrinology 17105.

Childs AJ, Cowan G, Kinnell HL, Anderson RA \& Saunders PT 2011 Retinoic acid signalling and the control of meiotic entry in the human fetal gonad. PLOS ONE 6 e20249. (https://doi.org/10.1371/journal.pone.0020249)

Crichton JH, Playfoot CJ, Maclennan M, Read D, Cooke HJ \& Adams IR 2017 Tex19.1 promotes Spo11-dependent meiotic recombination in mouse spermatocytes. PLoS Genetics 13 e1006904. (https://doi. org/10.1371/journal.pgen.1006904)

Crichton JH, Read D \& Adams IR 2018 Defects in meiotic recombination delay progression through pachytene in Tex19.1(-/-) mouse spermatocytes. Chromosoma 127 437-459. (https://doi.org/10.1007/ s00412-018-0674-9)

Deshong AJ, Ye AL, Lamelza P \& Bhalla N 2014 A quality control mechanism coordinates meiotic prophase events to promote crossover assurance. PLoS Genetics 10 e1004291. (https://doi.org/10.1371/ journal.pgen.1004291)

Dokshin GA, Baltus AE, Eppig JJ \& Page DC 2013 Oocyte differentiation is genetically dissociable from meiosis in mice. Nature Genetics $\mathbf{4 5}$ 877-883. (https://doi.org/10.1038/ng.2672)
Edson MA, Nagaraja AK \& Matzuk MM 2009 The mammalian ovary from genesis to revelation. Endocrine Reviews 30 624-712. (https://doi. org/10.1210/er.2009-0012)

Enders GC \& May 2nd JJ 1994 Developmentally regulated expression of a mouse germ cell nuclear antigen examined from embryonic day 11 to adult in male and female mice. Developmental Biology 163 331-340. (https://doi.org/10.1006/dbio.1994.1152)

Feng CW, Bowles J \& Koopman P 2014 Control of mammalian germ cell entry into meiosis. Molecular and Cellular Endocrinology 382 488-497. (https://doi.org/10.1016/j.mce.2013.09.026)

Frydman N, Poulain M, Arkoun B, Duquenne C, Tourpin S, Messiaen S, Habert R, Rouiller-Fabre V, Benachi A \& Livera G 2017 Human foetal ovary shares meiotic preventing factors with the developing testis. Human Reproduction 32 631-642. (https://doi.org/10.1093/humrep/ dew343)

Gondos B, Westergaard L \& Byskov AG 1986 Initiation of oogenesis in the human fetal ovary: ultrastructural and squash preparation study. American Journal of Obstetrics and Gynecology 155 189-195. (https:// doi.org/10.1016/0002-9378(86)90109-2)

Griswold MD, Bishop PD, Kim KH, Ping R, Siiteri JE \& Morales C 1989 Function of vitamin $\mathrm{A}$ in normal and synchronized seminiferous tubules. Annals of the New York Academy of Sciences 564 154-172. (https://doi. org/10.1111/j.1749-6632.1989.tb25895.x)

Griswold MD, Hogarth CA, Bowles J \& Koopman P 2012 Initiating meiosis: the case for retinoic acid. Biology of Reproduction 8635.

He Z, Henricksen LA, Wold MS \& Ingles CJ 1995 RPA involvement in the damage-recognition and incision steps of nucleotide excision repair. Nature 374 566-569. (https://doi.org/10.1038/374566a0)

Heller CG, Moore DJ \& Paulsen CA 1961 Suppression of spermatogenesis and chronic toxicity in men by a new series of bis(dichloroacetyl) diamines. Toxicology and Applied Pharmacology 3 1-11. (https://doi. org/10.1016/0041-008X(61)90002-3)

Hirshfield AN 1992 Heterogeneity of cell populations that contribute to the formation of primordial follicles in rats. Biology of Reproduction 47 466-472.

Hogarth CA, Evanoff R, Snyder E, Kent T, Mitchell D, Small C, Amory JK \& Griswold MD 2011 Suppression of Stra8 expression in the mouse gonad by WIN 18,446. Biology of Reproduction 84 957-965. (https:// doi.org/10.1095/biolreprod.110.088575)

Huang HF \& Hembree WC 1979 Spermatogenic response to vitamin A in vitamin A deficient rats. Biology of Reproduction 21 891-904. (https:// doi.org/10.1095/biolreprod21.4.891)

Koubova J, Menke DB, Zhou Q, Capel B, Griswold MD \& Page DC 2006 Retinoic acid regulates sex-specific timing of meiotic initiation in mice. PNAS 103 2474-2479. (https://doi.org/10.1073/pnas.0510813103)

Le Bouffant R, Guerquin MJ, Duquenne C, Frydman N, Coffigny $\mathbf{H}$, Rouiller-Fabre V, Frydman R, Habert R \& Livera G 2010 Meiosis initiation in the human ovary requires intrinsic retinoic acid synthesis. Human Reproduction 25 2579-2590. (https://doi.org/10.1093/humrep/ deq195)

Li H \& Clagett-Dame M 2009 Vitamin A deficiency blocks the initiation of meiosis of germ cells in the developing rat ovary in vivo. Biology of Reproduction 81 996-1001. (https://doi.org/10.1095/ biolreprod.109.078808)

Li H, Palczewski K, Baehr W \& Clagett-Dame M 2011 Vitamin A deficiency results in meiotic failure and accumulation of undifferentiated spermatogonia in prepubertal mouse testis. Biology of Reproduction $\mathbf{8 4}$ 336-341. (https://doi.org/10.1095/biolreprod.110.086157)

Lin Y, Gill ME, Koubova J \& Page DC 2008 Germ cell-intrinsic and -extrinsic factors govern meiotic initiation in mouse embryos. Science 322 1685-1687. (https://doi.org/10.1126/science.1166340)

Livak KJ \& Schmittgen TD 2001 Analysis of relative gene expression data using real-time quantitative PCR and the 2(-Delta Delta C(T)) method. Methods 25 402-408. (https://doi.org/10.1006/meth.2001.1262)

Menke DB, Koubova J \& Page DC 2003 Sexual differentiation of germ cells in XX mouse gonads occurs in an anterior-to-posterior wave. Developmental Biology 262 303-312. (https://doi.org/10.1016/S00121606(03)00391-9)

Minkina A, Lindeman RE, Gearhart MD, Chassot AA, Chaboissier MC, Ghyselinck NB, Bardwell VJ \& Zarkower D 2017 Retinoic acid signaling is dispensable for somatic development and function in the mammalian 
ovary. Developmental Biology 424 208-220. (https://doi.org/10.1016/j. ydbio.2017.02.015

Morales C \& Griswold MD 1987 Retinol-induced stage synchronization in seminiferous tubules of the rat. Endocrinology 121 432-434. (https://doi. org/10.1210/endo-121-1-432)

Morita Y \& Tilly JL 1999 Segregation of retinoic acid effects on fetal ovarian germ cell mitosis versus apoptosis by requirement for new macromolecular synthesis. Endocrinology 140 2696-2703. (https://doi. org/10.1210/endo.140.6.6826)

Mork L, Maatouk DM, Mcmahon JA, Guo JJ, Zhang P, Mcmahon AP \& Capel B 2012 Temporal differences in granulosa cell specification in the ovary reflect distinct follicle fates in mice. Biology of Reproduction 8637.

Motta PM, Makabe S \& Nottola SA 1997 The ultrastructure of human reproduction. I. The natural history of the female germ cell: origin, migration and differentiation inside the developing ovary. Human Reproduction Update 3 281-295. (https://doi.org/10.1093/ humupd/3.3.281)

Nilsson EE, Detzel C \& Skinner MK 2006 Platelet-derived growth factor modulates the primordial to primary follicle transition. Reproduction 131 1007-1015. (https://doi.org/10.1530/rep.1.00978)

Nilsson EE \& Skinner MK 2004 Kit ligand and basic fibroblast growth factor interactions in the induction of ovarian primordial to primary follicle transition. Molecular and Cellular Endocrinology 214 19-25. (https://doi. org/10.1016/j.mce.2003.12.001)

Paredes A, Garcia-Rudaz C, Kerr B, Tapia V, Dissen GA, Costa ME, Cornea A \& Ojeda SR 2005 Loss of synaptonemal complex protein-1, a synaptonemal complex protein, contributes to the initiation of follicular assembly in the developing rat ovary. Endocrinology 146 5267-5277. (https://doi.org/10.1210/en.2005-0965)

Parrott JA \& Skinner MK 1999 Kit-ligand/stem cell factor induces primordial follicle development and initiates folliculogenesis. Endocrinology $\mathbf{1 4 0}$ 4262-4271. (https://doi.org/10.1210/endo.140.9.6994)

Pepling ME 2006 From primordial germ cell to primordial follicle: mammalian female germ cell development. Genesis 44 622-632.

Pepling ME \& Spradling AC 2001 Mouse ovarian germ cell cysts undergo programmed breakdown to form primordial follicles. Developmental Biology 234 339-351. (https://doi.org/10.1006/dbio.2001.0269)

Pesce M, Wang X, Wolgemuth DJ \& Scholer H 1998 Differential expression of the Oct-4 transcription factor during mouse germ cell differentiation. Mechanisms of Deveopment 71 89-98.

Peters AH, Plug AW, Van Vugt MJ \& De Boer P 1997 A drying-down technique for the spreading of mammalian meiocytes from the male and female germline. Chromosome Research 5 66-68. (https://doi. org/10.1023/A:1018445520117)

Polani PE \& Crolla JA 1991 A test of the production line hypothesis of mammalian oogenesis. Human Genetics 88 64-70. (https://doi. org/10.1007/BF00204931)

Ramakers C, Ruijter J, Deprez R \& Moorman A 2003 Assumption-free analysis of quantitative real-time polymerase chain reaction (PCR) data. Neuroscience Letters 339 62-66. (https://doi.org/10.1016/S03043940(02)01423-4)
Rosario R, Crichton JH, Stewart HL, Childs AJ, Adams IR \& Anderson RA 2019 Dazl determines primordial follicle formation through the translational regulation of Tex14. FASEB Journal 33 14221-14233. (https://doi.org/10.1096/fj.201901247R)

Rowsey R, Gruhn J, Broman KW, Hunt PA \& Hassold T 2014 Examining variation in recombination levels in the human female: a test of the production-line hypothesis. American Journal of Human Genetics 95 108-112. (https://doi.org/10.1016/j.ajhg.2014.06.008)

Shi B, Xue J, Yin H, Guo R, Luo M, Ye L, Shi Q, Huang X, Liu M, Sha J et al. 2019 Dual functions for the ssDNA-binding protein RPA in meiotic recombination. PLoS Genetics 15 e1007952. (https://doi.org/10.1371/ journal.pgen.1007952)

Speed RM \& Chandley AC 1983 Meiosis in the foetal mouse ovary. II. Oocyte development and age-related aneuploidy. Does a production line exist? Chromosoma 88 184-189. (https://doi.org/10.1007/BF00285618)

Stefansdottir A, Johnston ZC, Powles-Glover N, Anderson RA, Adams IR \& Spears N 2016 Etoposide damages female germ cells in the developing ovary. BMC Cancer 16 482. (https://doi.org/10.1186/ s12885-016-2505-9)

Van Den Bergen JA, Miles DC, Sinclair AH \& Western PS 2009 Normalizing gene expression levels in mouse fetal germ cells. Biology of Reproduction 81 362-370.

Van Pelt AM \& De Rooij DG 1991 Retinoic acid is able to reinitiate spermatogenesis in vitamin A-deficient rats and high replicate doses support the full development of spermatogenic cells. Endocrinology 128 697-704. (https://doi.org/10.1210/endo-128-2-697)

Vandesompele J, De Preter K, Pattyn F, Poppe B, Van Roy N, De Paepe A \& Speleman F 2002 Accurate normalization of real-time quantitative RT-PCR data by geometric averaging of multiple internal control genes. Genome Biology 3 1-12.

Yin Y, Donlevy S \& Smolikove S 2016 Coordination of recombination with meiotic progression in the Caenorhabditis elegans germline by KIN-18, a TAO kinase that regulates the timing of MPK-1 signaling. Genetics 202 45-59. (https://doi.org/10.1534/genetics.115.177295)

Yu M, Xu Y, Li M, Li D, Lu Y, Yu D \& Du W 2018 Bisphenol A accelerates meiotic progression in embryonic chickens via the estrogen receptor beta signaling pathway. General and Comparative Endocrinology 259 66-75. (https://doi.org/10.1016/j.ygcen.2017.11.004)

Zaneveld LJ \& Waller DP 1989 Nonhormonal mediation of male reproductive tract damage: data from contraceptive drug research. Progress in Clinical and Biological Research 302 129-149; discussion 150-156.

Received 21 April 2020

First decision 27 May 2020

Revised manuscript received 29 May 2020

Accepted 8 June 2020 